\title{
Editorial
}

\section{Lessons from the Practice of Household Surveys ${ }^{[1]}$}

There is an increasing tendency to use questionnaire surveys in the research of Chinese organization and management. For 2005 alone, a web search generates several hundreds of Chinese language reports and articles based on current and past surveys of various organizations. Many of these surveys, however, lack documentation to show how they were implemented. Following a more careful reading of the materials from three well-known survey series of enterprises ${ }^{[2]}$, one still has concerns about survey quality, namely the validity and reliability of survey instruments, sample representativeness, and response biases as influenced by internal and external factors of the survey contexts.

In this essay, I do not evaluate the quality of organizational surveys, since my limited experience with two such surveys offers no systematic understanding of them. I have, instead, conducted many more household surveys, whose problems and analyses might give hints on how to obtain and improve the quality of organizational surveys. Although households and organizations are two different kinds of units of analysis, survey problems may commonly be associated with (1) sampling, (2) survey implementation, and (3) quality control of interviews. ${ }^{[3]}$ To elaborate on each of these three components of the household survey, I will base my discussion on the Chinese General Social Survey (CGSS), an annual household survey of the country that my sociology colleagues and I have conducted since 2003. At the end of this essay, I will draw from our household survey experiences a few lessons for organizational survey researchers.

\section{SAMPLING STRATEGIES AND PROBLEMS}

Household registration, street tracks, and the population census are the three possible frames from which to sample households, and all have drawbacks. Many sociologists use the first option in their local surveys; the CGSS uses the second and third options in its annual national surveys. Whichever option is taken, sampling communities, households, and respondents raises fresh challenges in a situation that is radically changing, such as that in China. 


\section{Household Registration}

Household registration, or the 'hukou' system, is now widely known to international researchers of China (Cheng and Selden, 1994). Local police in the urban districts and rural townships keep an up-to-date record of the fertility, mortality, migration, and demographic characteristics of permanent residents in the localities under their jurisdiction, thus providing the most reliable sampling frame for permanent residents. In the 1980s and early 1990s, city-based surveys by Chinese and non-Chinese social scientists relied on the household registration to successfully sample households and respondents (Bian, 2002; Tang, 2003). However, its records on temporary residents are much less reliable. Based on visits to a few local police offices in Guangzhou city where migrants have arrived in high numbers, the CGSS group obtained an estimate that, in 2003 , at best only $50 \%$ of the migrants were recorded by local police. Apparently then, household registration should not be the first choice of frame when sampling households and respondents in a radically mobile community, urban or rural.

\section{Street Tracks}

Sampling through the mapping of street tracks is a standard method of household survey in Western countries. It has recently become popular among survey researchers of urban China because of its promise to reach a representative sample of both permanent residents and migrants (Treiman et al., 2005). However, its weaknesses are obvious. Technical details aside (Liang, 2004), the method was developed in advanced countries in the West and assumes the existence of completely numbered residences that are well organized. This does not reflect Chinese reality: mutliunit courtyards with a single gate number, temporary dwellings without house numbers, self-made home splits with no registration, and one unit with multiple single-person households are fairly common residential arrangements in Chinese cities ( $\mathrm{Li}, 2004)$. Ironically, migrants are most likely to be found in one of these types of residence! Thus, the use of this method is labour-intensive and great care must be taken when drawing households in communities of complex construction. As a result, training, implementation, and monitoring costs increase.

\section{Population Gensus}

The population census offers the comparatively best, though not necessarily complete, record of households and addresses at the census year. Migrants may be seriously under-reported in the census, however; the estimated counting errors of the 2000 census are due largely to the under-reporting of migrants (Zhang and Cui, 2004). Another problem is the outdating of the census records caused by rapid changes of residents and homes; the impact of which is great in a radically chang- 
ing country like China. To a non-governmental user, the most acute problem is the restricted access to census records. The CGSS project was granted special permission to use the 2000 census to draw a multi-stage probability sample of urban and rural households. While working in the office, the computerized census file made it possible to generate sets of random samples to satisfy design requirements. Out in the field, however, we met challenges at all three levels of sampling implementation: communities, households, and respondents.

At the community level, the main challenge was that a census-recognized community might have disappeared due to city construction or residential reorganization. While nearly all cities have continuously had construction projects since the early 1990s, the Department of Civil Services (min zheng bu) administered a thorough residential reorganization in the country around 2002. For example, in 2003, Shanghai's 5 out of 40 (or 1/8) initially sampled neighbourhood committees were no longer existent, and in 2005 , Tianjin's $40 \%$ of the sampled neighbourhoods had disappeared or reorganized. Our solution to this challenge was to prepare replacement samples. In practice, we drew three separate random samples of communities (neighbourhood committees and rural villages): one served as the working sample and the other two as the reserve and replacement samples. Our utilization of replacement samples was $10 \%$ and $15 \%$ in the 2003 and 2005 surveys, respectively.

At the household level, there were two main challenges. The first was to locate a sampled household by matching the recorded address to the actual one. In 2003, we sampled households from the census-recognized addresses and found a signifcant number of unmatched addresses, due to the outdating or inaccuracy of the census records. To minimize the trouble, in 2005 we applied the mapping method to sample households (within the neighbourhoods and villages that were selected by using the census as the sampling frame), making the sample closer to the actual distribution of households of the year. The second challenge was in actually entering the sampled households to conduct interviews. Households generally do not welcome any survey, due to frequent marketing surveys, the altruistic nature of providing personal information, decreased leisure time, and distrust of strangers in a highly commercialized and unsafe society. The two well-known household survey series in Chinese society, the Hong Kong Social Indicators Survey (since 1990) and Taiwan Social Change Survey (since 1985), have had a response rate in the neighbourhood of $40-50 \%$. The CGSS had a response rate of $77 \%$ in 2003 and $41 \%$ in 2005 (both for urban areas), the difference being generated mostly by the assistance of local government staffers to help introduce the interviewers to the selected households in 2003. For those households for which no one answered the door, we required interviewers to make three tries at different times and days before characterizing them as 'empty households' to drop.

At the respondent level, beside the normal challenge of using the Kish grid $^{[t]}$ to ensure the random selection of a respondent, we faced the special challenge of 
'person-hukou separation' ( $r e n$ hu fen li). This is a situation where a person lives in a different residence from the one registered in the census. This occurs when a household member is absent through marriage, work, or relocation, or when a non-member has moved in long term without changing his/her permanent residence registration. Since we used the census records to draw households in our 2003 survey, we found about $10 \%$ of person-hukou separation households. This problem was eliminated in 2005 when we sampled households through the mapping of street tracks and households, a method that ignores hukou and recognizes residential actuality.

\section{THREE SYSTEMS OF SURVEY IMPLEMENTATION}

A scholar-led household survey can be implemented through one of the three systems of organization available in China: a scholarly network, a government organization, or a marketing survey firm. Each choice has obvious strengths and weaknesses as I shall describe below, but it is not feasible to combine these systems to implement a survey as that would generate serious conflicts in work authority and financial arrangements. To experiment and compare, the CGSS used each one of these systems in its first three years of annual surveys in turn. Thus, what I describe here is empirical knowledge gained from our 2003, 2004, and 2005 practices.

In 2003, we formed a scholarly network to conduct the CGSS. Scholarly networks are the most convenient system as scholars collaborate to empower their students to conduct surveys by themselves. This has been a standard practice in local surveys conducted by Chinese sociologists for three good reasons: it is an opportunity for scholars to train their students in social survey taking; students are hired as interviewers for a below-market price; the assistance of local governments can be obtained through network ties to help overcome difficulties in survey implementation. Because participating scholars are usually project collaborators, they are principals rather than agents, thus allowing for effective control to ensure survey quality. To capitalize on these strengths, the CGSS identified leading Chinese sociology programmes and invited their leaders to be regional participants and collaborators in the 2003 survey. From these programmes teachers and students were sent to conduct the survey in their regions. The serious drawback of using the scholarly networks, however, is the high consumption of time and energy of the Principle Investigator, his collaborators, and other scholars and students in labour-intensive survey interviews. To set against this, valuable first-hand knowledge was gained about conducting a national survey in a country of tremendous regional variation. This knowledge was indispensable when we revised our survey design and advised others on how to conduct later CGSS surveys.

In 2004, we turned to a government organization of high capacity to implement the CGSS survey. For this, we constructed the questionnaire independently 
and developed a contract with the government organization to carry out household interviews. This model follows the well-known Chinese Household Income Project, whose 1988 and 1995 datasets and scholarly publications have been circulated internationally (Khan and Riskin, 1998). Because the survey work was delegated to the government organization, the CGSS Principle Investigator and his collaborators had the benefit of not getting involved in survey implementation, just 'waiting for the data to come out' - so to speak, providing a substantial workload benefit. The drawback, however, was tremendous as the Principle Investigator and his collaborators lost touch with the details of such fieldwork as household sampling, respondent selection, and questionnaire interviews, thus lacking any measure of quality control over the survey implementation. The government organization had its own measures of quality control, but due to poor recording processes and sampling and interviewing procedures, these measures were opaque to the CGSS office.

We used a marketing survey firm to conduct the 2005 CGSS. Marketing survey firms are profit driven, and therefore we paid a significantly higher survey price in 2005 than in 2003 and 2004, but justified the cost in two ways. First, we openly discussed our design and generated clear standards by which to screen and select the best marketing survey firm. The selected marketing survey firm in turn provided a detailed plan of implementation from which to develop a business contract. Second, this contract specified a reasonable set of measures that we adopted to ensure the quality of household sampling and questionnaire interviews. These measures included our discretion to oversee the field work in any locality, to require a detailed recording of successful and failed household visits, to request mid-term and term-end reports of the interviewing results, and to hire a 'third company' to exercise quality control on our behalf. Increased competition among marketing survey firms put us in this advantageous position. While marketing survey firms in advanced countries may not conduct academic projects (which would be conducted by non-profit organizations), they are experienced at these kinds of projects in post-Communist countries like Russia and China (Gerber, 2002). Indeed, many survey firms were established by people with an academic background. Top marketing survey firms in China all have good connections to government authority and access to sources of official data.

Marketing survey firms may not work well for all the projects and certainly not for all clients. The firms must be familiar with the kinds of scholarly surveys under negotiation or should not be chosen to conduct them. In negotiation and implementation stages, the firms can be as opportunistic as they want to be, asking for more and doing less if the clients are inexperienced survey researchers or are not used to dealing with marketing firms. On the part of scholar clients, good negotiation must be backed by solid knowledge about the details of the entire process of survey implementation in the Chinese context. More important is to exercise high quality control measures, not just to let the chosen firm do what the contract 
requires, but also to have an experienced coordinator who will be in touch with the firm on a daily or weekly basis while the survey is being carried out. Problems must be identified quickly and resolved immediately. With these checks in place, the firm can deliver satisfactory results. One measure of the survey quality is the proportion of no answers to the general questions that all respondents must answer. Out of 41 common questions our urban respondents of the 2003 and 2005 CGSSs had, respectively, $1.9 \%$ and $1.7 \%$ of the questions were left unanswered. By this indicator alone, the marketing survey firm we hired produced results as good as the scholarly networks.

\section{QUALITY GONTROL MEASURES}

Quality control is about disciplining, monitoring, and adjusting the behaviour of interviewers in order to ensure the quality of the survey. Obviously, effective quality control is the key to success of any survey. Through first-hand experience, my colleagues and I developed a set of quality control measures that we applied to the 2003 CGSS. This experience and knowledge was also helpful during our contract negotiations with the government organization and especially marketing survey firms for the 2004 and 2005 CGSS, respectively. I describe these measures as they are applied to the pre-study, in-study, and post-study stages of the survey.

\section{Pre-study Quality Control Measures}

Pre-study measures of quality control include the development of an interviewer manual, which specifies all standards and procedures of a complete interview, and the training of interviewers. Because our CGSS is a national survey that is conducted by regional teams, our interviewer training was conducted at two levels: the first was to train regional supervisors, and the second was for the regional supervisors to train field supervisors and interviewers within the region. This 2level training format applied to every year's survey. At each level, trainees were requested to get acquainted with the questionnaire, to be well informed of items that needed special care, to conduct experimental interviews among trainees themselves, and to exchange experiences and skills after a couple of real-setting interviews conducted during the pilot study. Interviewer selection was made after the whole training process had been complete.

\section{In-study Quality Control Measures}

In-study measures of quality control are meant to reduce erroneous responses that emerge because of the misconduct of the interviewer. Erroneous responses can be caused by an interviewer who is unfaithful to the wording of the printed question, who is biased toward a style of questioning that misleads the respondent, who is 
careless in recording, or who, most disastrously, is cheating. A textbook solution (Fowler and Mangione, 1990) to identify and minimize these errors is the standardization of questionnaire items, which we adopted in our CGSS questionnaires. Above and beyond that, our lengthy survey experience informed us that a timely and adequate amount of onsite supervision from the field supervisor is the key to a quality interview. Supervision is provided during the checking of each completed questionnaire on site immediately after the interview. Not until the completed questionnaire passes the standard does the supervisor supply another questionnaire for the next interview. The onsite checking is aimed at identifying unclear markings, missed items, questionable answers, and dubious patterns of response. Identified errors need to be corrected right away, and revisits to respondents are to be arranged when serious errors are found. Onsite checking of the first three interviews is most important as the interviewer can learn a great deal that can be applied to later interviews. Double-checking is exercised by an upper-level supervisor at the end of the interview day or the following day.

\section{Post-study Quality Control Measures}

Post-study measures of quality control help to determine if an interviewer passes the threshold of being a quality interviewer. Here, the threshold is defined as the middle point, plus and minus one or two standard deviations, between 'good' and 'poor' interviews, and it is determined by looking at six indicators of what a poor completion of the questionnaire interview should be: (1) a large number of items unanswered; (2) a large number of 'don't know' and 'no answer' items; (3) a large number of 'check all that apply' questions to which only one answer is checked; (4) a large number of 'others' checked to multiple-choice questions; (5) a large number of skips to contingency questions; and (6) homogenous answers to attitude questions that are stated in a pattern of changing directions. These problems can be identified by analyzing the survey data, and the results are used to evaluate the quality of interviewers (student or professional interviewers that are affiliated with the project organization). To illustrate, Figure 1 presents two measures of post-study quality control from our 2003 GGSS. My purpose here is not to identify good or poor interviewers. Rather, the results are generated to show the overall quality of our CGSS surveys.

In the 2003 CGSS, about $90 \%$ of the interviews were conducted by student interviewers from our collaborating sociology programmes, and the additional $10 \%$ by interviewers hired from the communities of the survey sites (mostly in Guangdong province and Shanghai city). Many of these interviewers stayed on the job throughout the survey, while a small number of interviewers (mostly onsite hires) discontinued after a few completions. We recorded each interviewer's completions, and the data from the horizontal axis of Figure 1, termed 'interviewing sequencing'. The vertical axis is about the interviewer's effort-effectiveness, mea- 


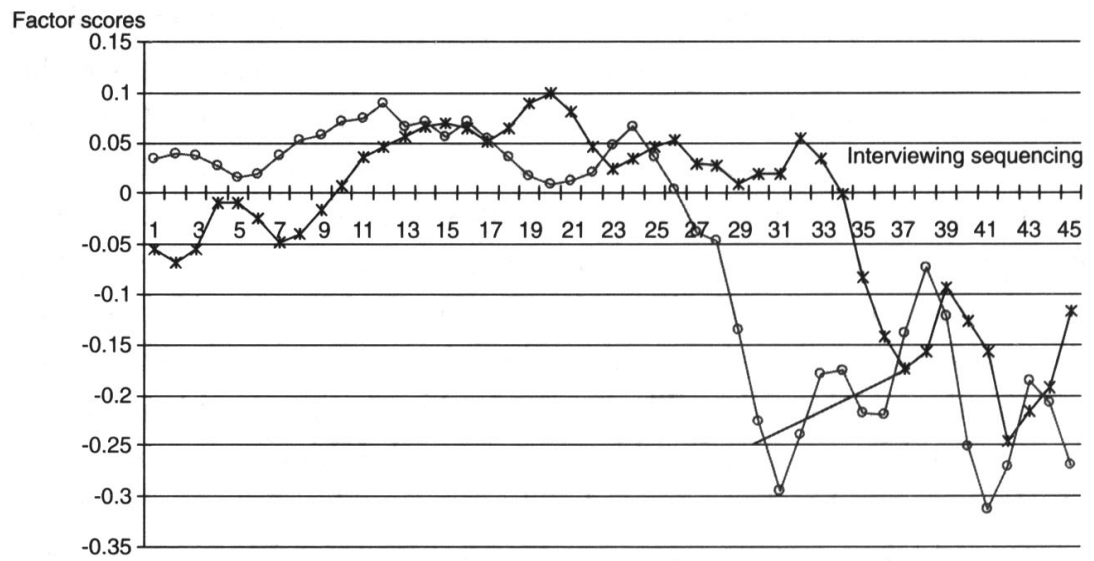

Figure 1. Interviewer effects on data quality

Note: Unanswered questions (line with $\mathbf{x}$ ) and Precision (line with o) by Interviewing Sequencing, 2003 Chinese General Social Survey.

sured by two quality control variables: number of unanswered questions and the degree of precision. These two variables have been transformed into standardized scales required in order to display the results in the same figure. Below I interpret each term in turn while presenting the results.

A question may be left unanswered when it is not applicable to the respondent. However, if an interviewer has many completed questionnaires in which signifcantly higher numbers of questions are left unanswered, this is an indication of a potentially poor interviewer. Out of 692 questions in the 2003 questionnaire, we identified 165 'general questions' which respondents generally must answer and are expected not to skip. These are about respondents' characteristics (age, gender, education, etc.), personal and family situations (income, durable goods possession, etc.), and behaviours (consumption, time spending, etc.). Out of 165 questions, on average 5.8 (or $3.5 \%$ ) questions were left unanswered. This indicates a very high quality survey in terms of unanswered questions. Many of these unanswered questions emerged before the fourth interview but significantly more emerged after the thirty-fourth interview, as indicated in Figure 1 (a factor score of 0 means average, greater than 0 means fewer unanswered questions, and less than 0 means more unanswered questions). Clearly, interviewers' increased familiarity with the questionnaire and improved interviewing skill paid off until interviewers gave up their efforts when a great number of such interviews had made them exhausted, careless, or ignorant.

The factor marked 'precision' was generated by measuring the pattern of responses to a series of attitude questions that are stated in a manner of changing directions: positive statements are mixed with negative statements. A 'precise' pattern of responses is that answers recognize the direction of the statements and vary from 'highly agreeable' to 'definitely not agreeable', and an 'imprecise' 
pattern of responses ignores the directions and stays with one point on a scale of varying answers. As shown in Figure 1, the precision is quite high up to the twentysixth interview, and it drops linearly from the twenty-seventh interview onward. Combining the two findings on unanswered questions and the precision of responses, we recommend no interviewer to be allowed to conduct more than 25 interviews using the same questionnaire.

\section{GONGLUDING REMARKS}

What can organizational survey researchers learn from the experience of the Chinese General Social Survey? On sampling, one is to be reminded of the population of targeted organizations for research and its optional frames from which to sample. To my knowledge, some researchers sample organizations from the Yellow Pages, others from the government registration, and still others take convenient samples without a sampling frame. The National Organization Survey project in the United States sampled organizations through the respondents in the General Social Survey, in which respondents were asked to give the names of their current workplaces. This sampling strategy has not been practiced in China. These and other sampling strategies may have serious implications for or against sample representativeness that ought to be taken into account at the stage of sample design and model estimation. To carry out sampling in the field, one must also be alert to new challenges that arise in an era of radical change in both economy and society. For example, some organizations have more than one name, others have a name but no place of operation, and still others register in one place while operating elsewhere. How to sample organizations in these situations? What strategies are required to enter a sampled organization in order to proceed with the survey?

On survey implementation, one should compare the strengths and weaknesses of different systems of organization and the choice is to be justified by the goal and resources of the survey project. Scholarly networks are most convenient but they require the full involvement of the scholars themselves. Government organization is cost efficient and demands little involvement of the scholars in the implementation of the survey, but the implementation is not as well documented as required to meet the standards of a scholar-led survey. Marketing survey firms, though profit driven, can meet a high quality standard as far as the survey principals work out a detailed contract that includes quality control measures. If the survey principals have a large survey project (a large budget), they are likely to be in a buyer's market, and, consequently, a favourable contract will be secured.

On quality control measures, household survey researchers pay a great deal of attention to the behaviour of interviewers. Measures of pre-study control and instudy control are absolutely necessary in household surveys, and I applied them, with a good deal of success, to my 2003 survey of 830 enterprises in the Pearl River Delta region. Post-study measures are useful to evaluate the overall quality 
of interviewers, as well as that of the survey. The 2003 CGSS has a very high quality for having both an extremely low number of unanswered questions and a fairly high degree of precision of responses to attitude questions. But we also found that when interviewers did more than 25 interviews, the quality began to decline. Such a tendency may not be repeated in organizational survey projects that have a smaller sample size than household surveys. After how many interviews do interviewers begin to be exhausted, careless, or ignorant of the interviewing standards in an organizational survey? This is a question to be answered by further research and experience.

Organizational researchers have favoured a few other survey methods that I did not discuss in my essay. These include mail surveys of organizational leaders, surveys of employees within organizations, and, most recently, the use of the internet to collect public or primary data in China. The internet is increasingly a source of good and timely data. Since anybody can put any kind of information on the internet, an assessment of the nature, scope, and quality of the data is necessary before they can be analyzed for research purposes. The internet may not be a good channel to conduct surveys, however. To assess the extent to which the internet might serve a purpose for the CGSS, a Beijing sociologist from our group attempted to conduct a survey of a targeted group of people through the internet. He invented a number of procedures to screen internet respondents (just like screening household members for their eligibility to serve as a respondent). Yet he found that the screened respondents are skewed toward 'internet playboys', who committed a series of 'crimes' such as cheating on personal characteristics, fake answers to behavioural and attitude questions, and multiple entries into the survey. Surveys through the internet seem to have a long way to go before they can be a reliable method of real data collection.

Surveys of employees within organizations are fairly comparable to household surveys in terms of the structure of sampling units. Whereas a household survey has multi-level sampling units like city district, neighbourhood, household, and respondent, a survey of employees in organizations must go through the levels of industry, organization, and division before selecting and interviewing employees. Thus, lessons from household surveys about sampling, survey implementation, and quality control members would apply directly to surveys of employees in organizations. A practical concern with surveys of employees in organizations is the extent to which selection is affected by convenience. A convenient sample can produce 'good' results, but they are neither interpretable nor generalizable simply because the sampling error of the distribution of respondents is unknown. Also, when the survey is conducted in groups, which is usually the case, peer pressure and group conformity may be influential, and thus response biases should be assessed and estimated.

Mail surveys are intended for educated respondents and they are frequently used in advanced societies and communities. Though not adequate for household 
surveys in China whose respondents vary tremendously in level of education, mail surveys are a good method when surveying organizational leaders. If the survey questionnaire is simple, and if the survey has a legitimate sponsor with a good reputation or high authority, a good proportion of the organizational leaders would answer the questionnaires with care and on a timely basis. But a low response rate is always expected from any mail survey, and therefore sample representativeness is a concern and should be addressed before the data can be analyzed. As a poststudy effort, the quality control measures from household surveys that I discussed in this essay may be useful to assess the quality of responses provided by organizational leaders from mail surveys. This assessment would include dividing the returned questionnaires into two or multiple groups through which to test hypotheses about response biases.

Both household and organization surveys in China are a post-1980 phenomenon. Before then no survey of households or organizations was allowed as the Communist party-state had a monopoly over the economy and society. Post-1980 reforms have changed almost every aspect of Chinese economy and society. Sociologists conducted a good number of household surveys from which to accumulate 'local knowledge' about how best to collect survey data in a radically changing Chinese context (Bian, Li, and Cai, 2004; Bian, Tu, and So, 2001). Organization and management scholars traditionally favoured case studies, but researchers of Chinese organization and management now also have a great interest in survey data because these data are indispensable when testing research hypotheses about the structure, processes, behaviours, and outcomes of organizations. The survey is the most frequently used method in Chinese organization and management research $(\mathrm{Li}$ and Tsui, 2000). Our experience suggests that it needs to be improved in order to collect quality data in the radically changing Ghinese context. Management and Organization Review can serve as a platform for scholarly discussion and exchange about organizational surveys in specific and research methods in general.

\section{NOTES}

[1] The CGSS project described in the essay has been funded by a Central Allocation grant from the Research Grants Committee of the Hong Kong SAR government (CA03/04.HSS01). I am grateful to Li Lulu for his collaboration in the project, to Liang Yucheng for research assistance, and to Anne Tsui for helpful comments and suggestions on an earlier draft of this editorial essay.

[2] These are (1) the Chinese Entrepreneurs Survey Initiative (Zhongguo Qiye Jia Diaocha Xitong), which has been sponsored and organized by the Institute of Development of the State Council (Guowouyuan Fazhan Yanjiu Zhongxin) since 1993; (2) the Chinese Private Enterprise Surveys (Zhongguo Siying Qive Diaochao), which have been conducted by the Chinese Academy of Social Sciences with the sponsorship of the All China Alliance of Industrialists and Commercialists, having completed six surveys in 1993, 1995, 1997, 2000, 2002, and 2004; and (3) the National Statistical Bureau's Enterprise Survey Series, an annual survey of industrial enterprises since 1990.

[3] This essay is about the design and implementation of a survey based on personal interviews, not mail responses. The difference between mail and personal interview surveys is found in the implementation and quality control stages. The sampling considerations should be the same in both 
methods. See Dillman (2000) for procedures on using mail surveys, as well as on the use of the internet to collect data.

[4] The Kish grid is a method used to randomly select from all eligible household members a respondent to answer the questionnaire. The method lists all eligible household members on the provided Kish grid. Each row is marked with numbers that are used to select a respondent randomly.

\section{REFERENGES}

Bian, Y. (2002). Chinese social stratification and social mobility. Annual Review of Sociology, 28, 91-116.

Bian, Y., Tu, E. and So, A. (Eds.) (2001). Survey Research in Chinese Societies: Methods and Findings (Zhongguo Shehui de Diaocha Yanjü). Hong Kong: Oxford University Press.

Bian, Y., Li, L. and Cai, H. (Eds.) (2004). Social Survey Research in Practice: Chinese Experiences and Analyses (Shehui Diaocha Shïian: Zhongguo Jingyan yu Fenxi). Hong Kong: Oxford University Press.

Cheng, T. and Selden, M. (1994). The origins and social consequences of China's hukou system. China Quarterly, 139, 644-68.

Dillman, D. A. (2000). Mail and Internet Surveys: The Tailored Design Method. $\left(2^{\text {nd }}\right.$ Ed.). New York, NY: John Wiley \& Sons.

Fowler, F. Jr. and Mangione, T. W. (1990). Standardized Survey Interviewing: Minimizing Interviewer-related Errors. Newbury Park, CA: Sage Publications.

Gerber, T. P. (2002). Structural change and post-socialist stratification: Labor market transitions in contemporary Russia. American Sociological Review, 67, 629-59.

Khan, A. R. and Riskin, C. (1998). Income inequality and its distribution and growth of household income, 1988 to 1995. China Quarterly, 154, 221-53.

Li, Q. (2004). Where do migrants hide? Presented at the International Conference on China's Fifth Population Census, Beijing, April 20-3.

Li, J. T. and Tsui, A. S. (2000). Management and Organizations in the Chinese Context. London: McMillan Press.

Liang, Y. (2004). An assessment of sampling methods applied to social surveys in Chinese cities. In Bian, Y., Li, L. and Cai, H. (Eds.) Social Survey Research in Practice: Chinese Experience and Analysis. Hong Kong: Oxford University Press (pp. 63-84).

Tang, W. (2003). An introduction to survey research in urban China. Issues \& Studies, 38-39, 269-88.

Treiman, D. J., Mason, W. M., Lu, Y., Pan, Y., Qi, Y. and Song, S. (2005). Observations on the design and implementation of sample survey in China. Social Transformation in Chinese Societies, 1, 81-112.

Zhang, W. and Cui, H. (2004). An estimation of the accuracy of China's 2000 population census. Presented at the International Conference on China's Fifth Population Census, Beijing, April 20-3.

Yanjie Bian

Editor 